\title{
Discrete Spherical Harmonic Transforms of Nearly Equidistributed Global Data
}

\author{
Research article
}

\author{
J.A. Rod Blais*
}

Department of Geomatics Engineering, Pacific Institute for the Mathematical Sciences

University of Calgary, Calgary, AB, T2N 1N4

\begin{abstract}
:
Discrete Spherical Harmonic Transforms (SHTs) are commonly defined for equiangular grids on the sphere. However, when global array data exhibit near equidistributed patterns rather than equiangular grids, discrete SHTs require appropriate adaptations for analysis and synthesis. Computational efficiency and reliability impose structural constraints on possible equidistribution characteristics of data patterns such as for instance with Chebychev quadratures and Fast Fourier Transforms (FFTs). Following some general introduction to discrete SHTs and equidistributions on the sphere, equitriangular (near equiareal) lattices based on the octahedron and the icosahedron are introduced for SHT analysis and synthesis. The developed formulations are described and implemented using simulated data and geopotential models such as the Earth Geopotential Model EGM 2008. Comparative results for analysis and synthesis at different levels of resolution show the potential of the spherical equitriangular approach for geodetic and other applications with nearly equidistributed global data.
\end{abstract}

\section{Keywords:}

Fourier Transforms • Geocomputations • Geopotential Modeling • Spherical Harmonics

(c) Versita Warsaw and Springer-Verlag Berlin Heidelberg.

Received 26 April 2011; accepted 01 June 2011

\section{Introduction}

Spherical harmonic transforms are non-commutative Fouriertransforms on the sphere. These transforms are global as for any regional subdomain of the sphere, ordinary Fourier transforms can be used for spectral analysis and other applications. Discrete SHTs are most often formulated for equiangular grids with equispaced parallels for Chebychev quadratures and equispaced meridians for Fast Fourier Transform (FFT) applications. Non-equispaced parallels can be handled using least squares with much less computational efficiency than with Chebychev quadratures, see e.g. Colombo [1981], Sneeuw [1994], and Blais [2011] for details and references. However when global data are nearly equidistributed, the equian-

*E-mail: blais@ucalgary.ca gular strategies need to be reconsidered especially for spherical multiresolution analysis and synthesis.

From elementary geometry, the five Platonic (regular) polyhedra provide the only rigorous equispaced regular sets of points on the circumscribing sphere. Explicitly, the tetrahedron, the cube, the octahedron, the icosahedron and the dodecahedron are the only convex regular polyhedra. The well-known duality between the cube and the octahedron can be exploited in the sense that associating discrete values to the vertices of a cube is equivalent to associating those quantities with the face centres of the octahedron. Similarly for the vertices of the dodecahedron and the face centres of the icosahedron. The triangular faces of the octahedron and the icosahedron can be subdivided to generate a spherical quad-tree (or two-dimensional binary tree) structure of equal triangular projections onto the circumscribed sphere (see e.g. [Haagmans, 2000] and [Klees et al, 2001]). Such a strategy which has been extensively used with global environmental data

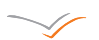

VERSITA 
[e.g. Fekete and Treinish, 1990] can be seen to be very closely compatible with the usual equiangular approach to discrete spherical harmonic transforms using Chebychev quadratures and least squares.

The approach of estimating spherical harmonic transforms using octahedron and icosahedron based triangulations can be shown to be appropriate to handle near-equiareal lattices of geodetic and similar data with fast decreasing power spectra (see e.g. [Rapp, 1989] and [Flury, 2006]). This is demonstrated using spherical harmonic synthesis and analysis simulations based on the Earth Geopotential Model EGM 2008 [Pavlis et al, 2008]. The limitations of the approach for data with slowly decreasing power spectra are also briefly discussed and general recommendations are included for practical applications.

\section{Spherical Harmonic Transforms}

The orthogonal or Fourier expansion of a function $f(\theta, \lambda)$ on the sphere $S^{2}$ is given by

$$
f(\theta, \lambda)=\sum_{n=0}^{\infty} \sum_{|m| \leq n} f_{n, m} Y_{n}^{m}(\theta, \lambda)
$$

using colatitude $\theta$ and longitude $\lambda$, where the basis functions $Y_{n}^{m}(\theta, \lambda)$ are called the spherical harmonics of degree $n$ and order $m$. The usual geodetic spherical harmonic formulation is given correspondingly as

$$
f(\theta, \lambda)=\sum_{n=0}^{\infty} \sum_{m=0}^{n}\left[\tilde{c}_{n m} \cos m \lambda+\tilde{s}_{n m} \sin m \lambda\right] \widetilde{P}_{n m}(\cos \theta)
$$

where

$$
\left\{\begin{array}{c}
\tilde{c}_{n m} \\
\tilde{s}_{n m}
\end{array}\right\}=\frac{1}{4 \pi} \int_{s^{2}} f(\theta, \lambda)\left\{\begin{array}{c}
\cos m \lambda \\
\sin m \lambda
\end{array}\right\} \widetilde{P}_{n m}(\cos \theta) d \sigma
$$

with the geodetically normalized Legendre functions $\widetilde{P}_{n m}(\cos \theta)$ expressed in terms of the usual spherical harmonics $Y_{n}^{m}(\theta, \lambda)$ (see e.g. [Heiskanen and Moritz, 1967] and [Blais and Provins, 2002] for details). The tilde " $\sim$ " is used herein to indicate the usual geodetic normalization.

Explicitly, using the geodetic formulation and convention, one has for synthesis with spherical band limit $N$

$$
f(\theta, \lambda)=\sum_{n=0}^{N-1} \sum_{m=0}^{n}\left[\tilde{c}_{n m} \cos m \lambda+\tilde{c}_{n m} \sin m \lambda\right] \widetilde{P}_{n m}(\cos \theta)
$$

and for analysis, using complex notation,

$$
\tilde{c}_{n m}+i \tilde{s}_{n m}=\frac{1}{4 \pi} \int_{0}^{2 \pi} \int_{0}^{\pi} f(\theta, \lambda)(\cos m \lambda+i \sin m \lambda) \widetilde{P}_{n m}(\cos \theta) \sin \theta d \theta d \lambda=\int_{0}^{\pi}\left[u_{m}(\theta)+i v_{m}(\theta)\right] \widetilde{P}_{n m}(\cos \theta) \sin \theta d \theta
$$

where

$$
u_{m}(\theta)+i v_{m}(\theta)=\frac{1}{4 \pi} \int_{0}^{2 \pi} f(\theta, \lambda(\cos m \lambda+i \sin m \lambda) d \lambda
$$

which is simply the parallel-wise Fourier transform of the spatial array data.

Using equiangular grids for degree $N$, the Chebychev Quadrature (CQ) is as follows

$$
\tilde{c}_{n m}^{\prime}+i \tilde{s}_{n m}^{\prime}=\sum_{j=0}^{2 N-1} q_{j}\left(u_{j m}+i v_{j m}\right) \widetilde{P}_{n m}\left(\cos \theta_{j}\right)
$$

with $u_{j m} \equiv u_{m}\left(\theta_{j}\right)$ and $v_{j m} \equiv v_{m}\left(\theta_{j}\right)$ (shifted) isolatitudes $\theta_{j}=\left(j+\frac{1}{2}\right) \pi / 2 N$ and CQ weights

VERSITA
$q_{j}=\frac{1}{N} \sin \left(\left(j+\frac{1}{2}\right) \pi / 2 N\right) \sum_{h=0}^{N-1} \frac{1}{2 h+1} \sin \left((2 h+1)\left(j+\frac{1}{2}\right) \pi / 2 N\right)$

with $q_{2 N-j}=q_{j}$ for $j=0,1, \ldots, N-1$ by hemispherical symmetry. These computations are roughly $\mathrm{O}\left(N^{3}\right)$ for degree $N$. More details about these Chebychev weights can be found in [Blais, 2011].

A Least-Squares (LS) formulation per order $m$ (for maximum degree $N)$ can also be used for spherical harmonic analysis:

$$
\sum_{n=m}^{N-1} \widetilde{P}_{n m}\left(\cos \theta_{j}\right)\left(\tilde{c}_{n m}^{\prime \prime}+i \tilde{S}_{n m}^{\prime \prime}\right)=u_{m}\left(\theta_{j}\right)+i v_{m}\left(\theta_{j}\right)
$$

with (shifted) isolatitudes $\theta_{j}=\left(j+\frac{1}{2}\right) \pi / N$ for $j=0,1, \ldots$, $N-1$, again taking advantage of hemispherical symmetry. The least-squares computations for $\tilde{c}_{n m}^{\prime \prime}+i \tilde{s}_{n m}^{\prime \prime}$ per order $\mathrm{m}$ are ob- 
viously very demanding and roughly $\mathrm{O}\left(\mathrm{N}^{4}\right)$. More details can be found in Blais [2011].

For some practical applications, equiangular grids are not appropriate for various reasons and some equidistribution alternatives are desirable. Moreover, in cases involving spherical convolutions such as filtering and multiresolution applications, the use of FFTs and SHTs are highly desirable for computational efficiency and reliability in large computations. Hence with appropriate constraints, equidistributed arrays of nodes are investigated as alternatives for equiangular grids for spherical harmonic analysis and synthesis.

\section{Equidistributions on the Sphere}

A spherical equiangular lattice of points obviously does not exhibit an equidistributed point set over the two-dimensional spherical surface. Equidistribution or uniform areal distribution of a point set is measured by discrepancy quantities which correspond to the supremum of cell point densities relative to the corresponding uniform densities. Considering the points or nodes $\xi_{n}: n=1, \ldots, N$ on the spherical surface, for an arbitrary cell $C$, discrepancy can be defined by

$$
\text { discrepancy }=\sup _{\text {all } C \in S^{2}} \frac{\sum_{n=1}^{N} X_{C}\left(\xi_{n}\right) / N}{\lim _{N \rightarrow \infty} \sum_{n=1}^{N} X_{C}\left(\xi_{n}\right) / N}
$$

in which $\chi_{C}\left(\xi_{n}\right)$ denotes the characteristic function for the cell C. There are numerous definitions of discrepancy in the literature but the simple preceding definition is sufficient to convey the conceptual idea for the sphere $S^{2}$. In more general contexts, it is known that for the s-dimensional cube, pseudo-random numbers can only achieve a discrepancy level of $(\log \log N)^{1 / 2} / N^{1 / 2}$ while it is possible to construct quasi-random (deterministic) sequences with discrepancy level of $(\log N)^{S} / N$ (see e.g. Morokoff and Caflisch [1994] for details and references). Notice that the dependency of the discrepancy on the dimension implies that quasi-random sequences are potentially advantageous over pseudo-random numbers in higher dimensions (see e.g. Blais and Zhang [2011]).

For spherical harmonic applications, Cui [1995], Cui and Freeden [1997] and Freeden [1998] discuss various node sequences of low discrepancy on the sphere. From a geometrical perspective, the vertices of regular polyhedra inscribed in the sphere obviously imply low discrepancy sequences, e.g. the tetrahedron, the cube, the octahedron, the icosahedron and the dodecahedron. Adaptations of these and other sequences due to Faure, Halton, Hammersley, Sobol, Niederreiter and van der Corput among others are well known in quasi Monte Carlo methods (see the preceding references). For spherical harmonic analysis and synthesis for multiresolution applications, different constraints are most important such as the hierarchical or pyramidal properties of the data structure (which imply that any level data set becomes a subset of all higher-level data sets) and the various requirements for SHTs using FFTs, Chebychev quadratures and least-squares formulations.

In the following, the equiangular approach will be adapted to densifications of the octahedron and icosahedron triangular faces projected onto the circumscribing sphere to achieve near equiareal triangulation structures. These quad-tree structures (implied by the partitioning of the triangular faces) have advantageous characteristics such as equilatitude subsets of equispaced points for quadratures and Fast Fourier computations. Furthermore, these configurations can readily be generated by applying a mask to the corresponding equiangular lattices of points. This is very advantageous for practical experimentation with and without any mask for comparative analysis and computational efficiency. Simulations will be carried out using the EGM 2008 spherical harmonic coefficients.

\section{Octahedron-Based Strategies}

An octahedron is a regular polyhedron with six vertices, eight (equitriangular) faces and twelve edges. Each triangular face can be subdivided into four equitriangular areas whose vertices can be projected onto the spherical surface for a higher level partition of the surface. This is the spherical quad-tree structure shown graphically in Figure 1. It is important to notice the octahedron is dual to the cube which has eight vertices, six (square) faces and twelve edges. Hence associating data values with the octahedron face centres is equivalent to associating data values with the vertices of a cube, and so on at higher levels of spherical partition. Using the cube and/or the octahedron, a near-equiareal partition of the (unit) sphere has been constructed and used extensively in the Healpix project [Gorski et al, 1998]. This strategy has proven very appropriate in astrophysical and related applications such as COBE and WMAP. One discrete gridding of the sphere goes as follows in colatitude $\theta$ by longitudes $\lambda$ :

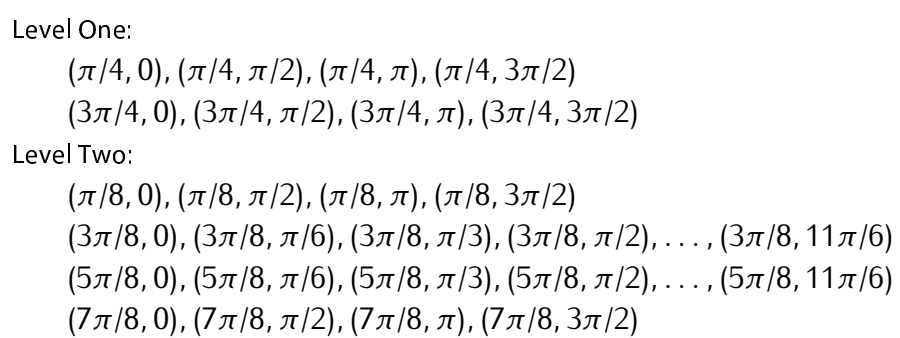

Level One: 


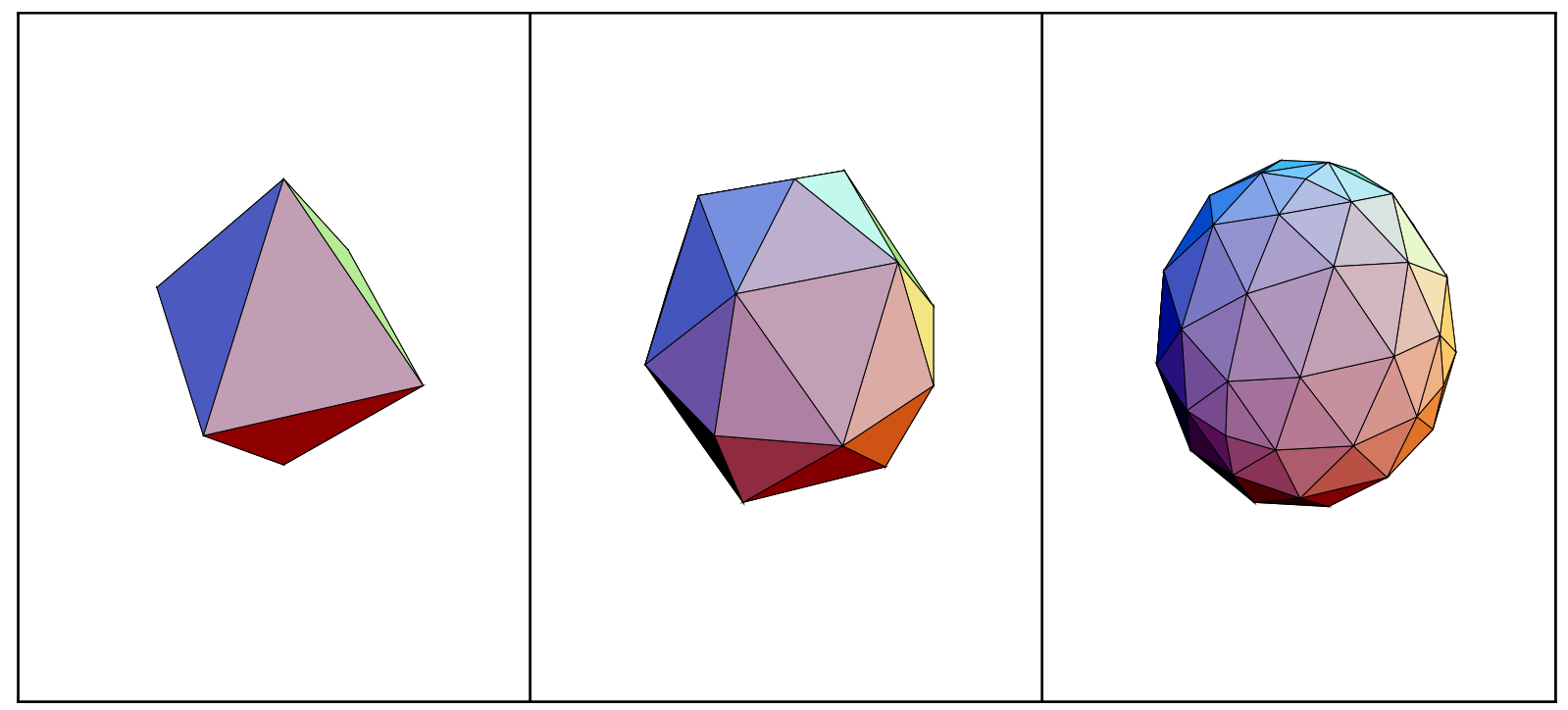

Figure 1. Octahedron and Spherical Quadtree Densification

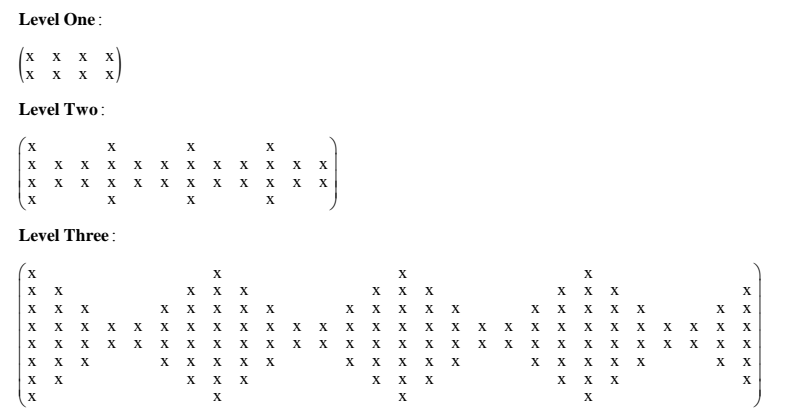

Figure 2. Octahedron Based Spherical Quad-tree Data Structure for Resolution Levels 1, 2 and 3

and so on for higher levels, as shown graphically in Figure 2. Notice that the latitudes have been recentered (at each level) to take advantage of the hemispherical symmetry in the computations. The triangular faces $\left(\Delta^{\prime} s\right)$ lead to a recursive near-equiareal partition of the spherical surface (see Figure 1) and a spherical quad-tree data structure:

$$
\begin{aligned}
& 2 \times(4) \Delta^{\prime} s \rightarrow 2 \times(4+12) \Delta^{\prime} s \rightarrow \\
& 2 \times(4+12+20+28) \Delta^{\prime} s \rightarrow \ldots
\end{aligned}
$$

with isogridlines and constant $\Delta \lambda$ thereon for FFT computations. Notice that such grid has overall dimensions $N \times 4(N-1)$ with $N$ even at each level, with exact hemispherical symmetry, which is compatible with the Chebychev quadrature and least-squares approaches discussed in the previous Section.

From Level One in Figure 2, the configuration is simply the same as an equiangular one giving a data matrix of two rows and four C columns. From Level Two in Figure 2, the configuration is different from the equiangular one with four rows and 12 columns with only 32 values out of the 48 elements. In general for Level $L$, one has only $2^{2 L+1}$ data values out of a regular matrix of $2^{L}$ rows and $4 \times\left(2^{L}-1\right)$ columns. Notice that the resulting data have a quad-tree data structure in both configurations for variable levels $L=1,2,3, \ldots$

\section{Icosahedron-Based Strategies}

An icosahedron is a regular polyhedron with twelve vertices, twenty (equitriangular) faces and thirty edges. Each triangularface can be subdivided into four equitriangular areas whose vertices can be projected onto the spherical surface for a higher level partition of the surface. This is the spherical quad-tree structure shown graphically in Figure 3. It is important to notice the icosahedron is dual to the dodecahedron which has twenty vertices, twelve (pentagonal) faces and thirty edges. Again this implies that associating data values with the icosahedron face centres is equivalent to associating data values with the vertices of a dodecahedron, and so on at higher levels of spherical partition. However, for exact hemispherical symmetry, the lower hemisphere has to be rotated clockwise or counterclockwise by an angle of $\pi / 5$. However, this offset is only really significant at Level 1 which is a special case when using geopotential and similar geodetic data because of the null spectral coefficients of degrees zero and one [Heiskanen and Moritz, 1967].

Based on the icosahedron, assuming an approximate hemispherical symmetry, the discrete gridding of the sphere goes as follows in colatitude $\theta$ by longitudes $\lambda$ : 


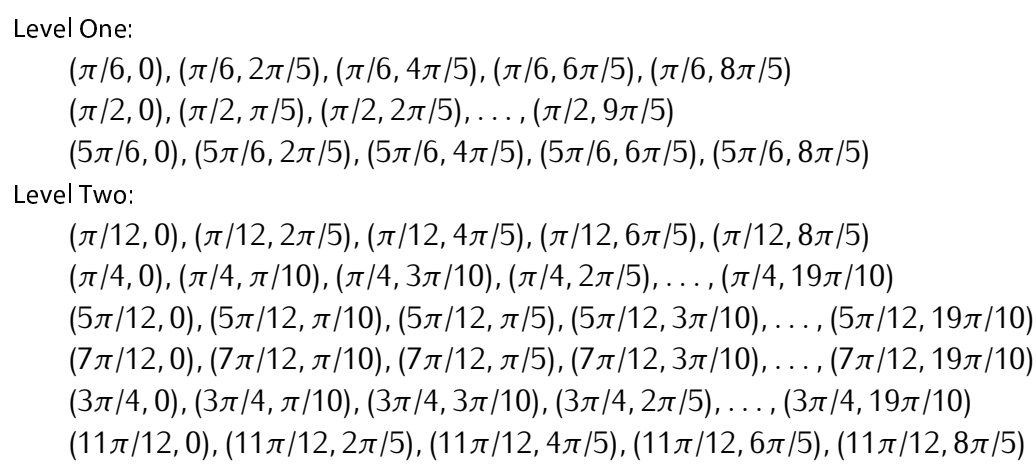

and so on for higher levels, as shown graphically in Figure 4. Again, notice that the latitudes have been recentered (at each level) to take advantage of the hemispherical symmetry in the computations. The triangular faces $\left(\Delta^{\prime} s\right)$ lead to a recursive nearequiareal partition of the spherical surface (see Figure 3 ) and a spherical quad-tree data structure:

$$
\begin{aligned}
& 2 \times(5)+10 \Delta^{\prime} s \rightarrow 2 \times(5+15)+10(4) \Delta^{\prime} s \rightarrow \\
& 2 \times(5+15)+\ldots \Delta^{\prime} s \rightarrow \ldots
\end{aligned}
$$

with isogridlines and constant $\Delta \lambda$ thereon for FFT computations. From Level One in Figure 4, the configuration exhibits a data matrix of three rows and ten columns with only 20 nodes out of the 30 elements. From Level Two in Figure 4, the configuration has six rows and 20 columns with only 80 values out of the 120 elements. In general for Level $L$, one has only $5 \times 2^{L+1}$ data values out of a regular matrix of $3 \times 2^{L-1}$ rows and $5 \times 2^{L}$ columns. Notice that the resulting icosahedron-based data have a quad-tree data structure in both configurations for variable Levels $L=1,2,3, \ldots$. but Level One is not compatible with the Chebychev quadrature as the number of rows is odd.

\section{Numerical Experimentation}

The latest Earth Geopotential Model EGM 2008 of maximum degree and order 2190, available from http://earth-info.nima.mil/ GandG/, can be considered as the most complete combined geopotential model for terrestrial applications such as geoid undulations, deflections of the vertical, etc. (see [Pavlis et al, 2008] for details). The EGM 2008 spectrum is well known to decrease rapidly with increasing degrees so that the high latitude gaps in the data matrices will be much less problematic than when using noise simulations with constant spectra (as done e.g. in [Blais, 2011]). Obviously, this is only significant in the analysis part of nearly equidistributed data as the synthesis is completely unaffected. The EGM 2008 spectrum has null coefficients of degrees zero and one as these are the so-called forbidden harmonics which is well-known in geodesy [Heiskanen and Moritz, 1967]. Such null coefficients obviously affect any low-level octahedral and icosahedral simulations with EGM 2008 and other similar geopotential data.
The spherical harmonic transform formulation does not obviously have any such constraint for degrees zero and one.

\subsection{Using the Octahedron-Based Triangulation Approach}

Using the data matrix templates corresponding to the octahedron shown in Figure 2, simulations have been carried out for the first 12 levels of octahedral triangulation using the Chebychev quadrature (CQ) and the first 11 levels using least squares (LS). Tables 1 and 2 show respectively the CQ and LS results in terms of spectral RMS (i.e. after synthesis and analysis) and spatial RMS (i.e. after a second synthesis) first, without mask (i.e. full equiangular grid) and second, with mask (i.e. equitriangular grid). The results corresponding to the full data matrices (without mask) are very stable and essentially of the same order of magnitude as for corresponding equiangular results with EGM 2008 given in Blais [2011]. The results corresponding to the octahedron-based data (i.e. applying the masks) are less accurate as expected but very stable numerically. The loss of accuracy with equidistributed data depends on the corresponding spectrum as the missing high latitude equiangular values affect the FFT results in the first part of the analysis.

\subsection{Using the Icosahedron-Based Triangulation Approach}

Using the data matrix templates corresponding to the icosahedron shown in Figure 3, simulations have been carried out for 10 levels of icosahedral triangulation using the Chebychev quadrature (CQ) and least squares (LS). Tables 3 and 4 show respectively the CQ and LS results in terms of spectral RMS (i.e. after synthesis and analysis) and spatial RMS (i.e. after a second synthesis) first, without mask (i.e. full equiangular grid) and second, with mask (i.e. equitriangular grid). The results corresponding to the full data matrices (without mask) are very stable and essentially of the same order of magnitude as for corresponding equiangular results with EGM 2008 given in Blais [2011]. The results corresponding to the icosahedron-based data (i.e. applying the masks) are much less accurate as expected but very stable numerically.

Notice that in Table 4, Level One with the $3 \times 10$ grid is not compatible with the CQ approach and the RMS values are trivial

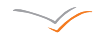

VERSITA 


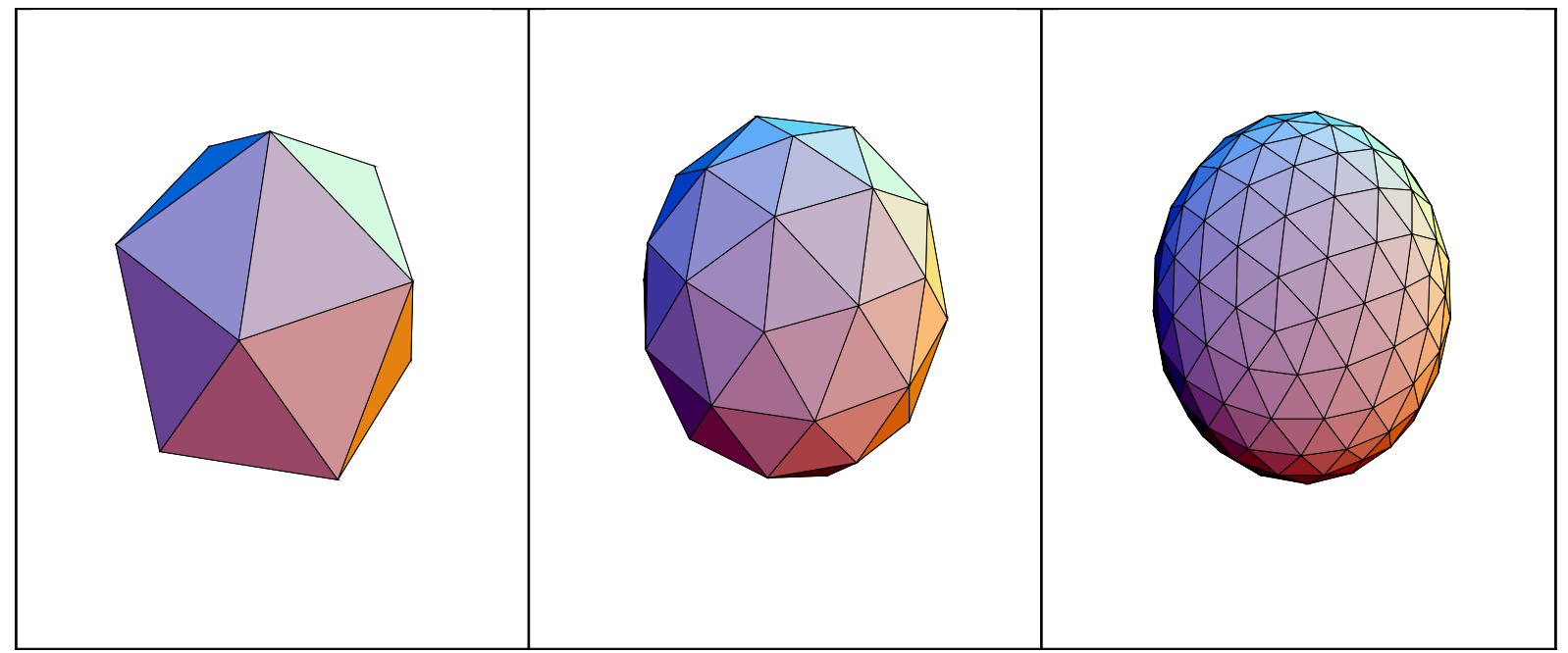

Figure 3. Icosahedron and Spherical Quad-Tree Densification

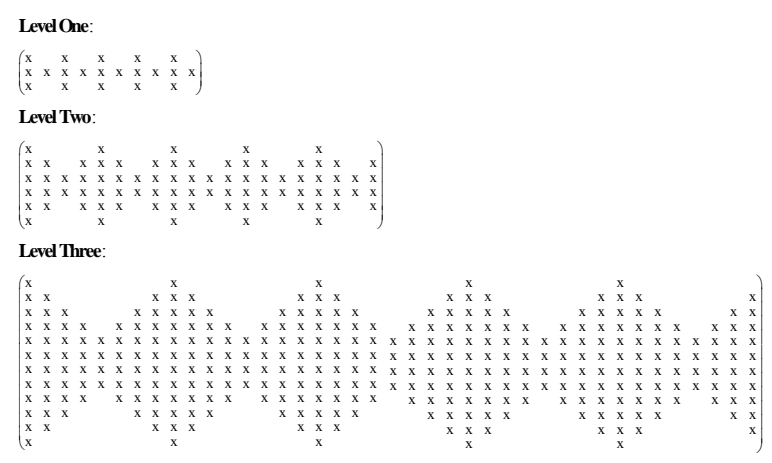

Figure 4. Icosahedron Based Spherical Quadtree Data Structure for Resolution Levels 1, 2 and 3

with LS because of the null spectral coefficients of degrees zero and one in EGM 2008.

\section{Concluding Remarks}

The equiangular grids which are conventional with discrete spherical harmonic transforms are not always appropriate in practice when the observational data are nearly equispaced or equidistributed on the sphere. Spherical quad-tree triangulation approaches using the octahedron and icosahedron triangular faces have been investigated for spherical harmonic analysis and synthesis. The modified SHT approach for multiple resolutions shows good accuracy and numerical stability with EGM 2008 and similar geodetic data with rapidly decreasing spectra. The high-latitude sparsity of data with such spherical equidistribution affects the estimation of high-degree spectral coefficients but the implications have been demonstrated to be quite acceptable with common geodetic data such as simulated using EGM 2008. In practice with noise-like data (i.e. with nearly constant spectra), following a preliminary analysis with near-equidistributed data, a synthesis on an equiangular grid may be required to refine the estimated spectral coefficients through a second analysis of the estimated equiangular grid values.

Notice that the approximate hemispherical symmetry assumed in the case of icosahedron-based simulated data gives analysis and synthesis results comparable with the octahedron-based data where the hemispherical symmetry is exact. Furthermore, such an approximation which is required for equilongitude patterns needed with CQ and LS appears to improve with higher levels of resolution at least in simulations using EGM 2008.

In conclusion, when dealing with approximately equidistributed geodetic and other similar data on the sphere, the equitriangular quad-tree approach based on the octahedron and the icosahedron provides an effective adaptation of the usual equiangular spherical harmonic transform for multiresolution analysis and synthesis. Furthermore, the computational efficiency of the modified SHTs is comparable to the previously documented SHT using CQ and LS.

\section{References}

Blais, J.A.R.: Discrete Spherical Harmonic Transforms for Equiangular Grids of Spatial and Spectral Data. Journal of Geodetic Science, Volume 1, Issue 1, 7 pages, 2011, Versita and Springer-Verlag.

Blais, J.A.R.: Optimal Spherical Triangulation for Global Multiresolution Analysis and Synthesis. Poster presentation at the 2007 Fall Meeting of the American Geophysical Union in San Francisco, CA.

\section{VERSITA}


Table 1. Spherical Analysis and Synthesis of 12 Resolution Levels of Octahedral Simulation Using the Chebychev Quadrature Formulation on the Unit Sphere

\begin{tabular}{lcccc}
\hline OCTAHEDRAL & \multicolumn{2}{c}{ CQ SHT of EGM2008 without mask } & \multicolumn{2}{c}{ CQ SHT of EGM2008 with mask } \\
\hline Level: Grid & Spectral RMS & Spatial RMS & Spectral RMS & Spatial RMS \\
\hline \hline 1: $2 \times 4$ & $0.000000 \mathrm{E}+00$ & $0.000000 \mathrm{E}+00$ & $0.000000 \mathrm{E}+00$ & $0.000000 \mathrm{E}+00$ \\
2: $4 \times 12$ & $0.000000 \mathrm{E}+00$ & $0.000000 \mathrm{E}+00$ & $0.000000 \mathrm{E}+00$ & $0.000000 \mathrm{E}+00$ \\
3: $8 \times 28$ & $5.015181 \mathrm{E}-22$ & $2.466575 \mathrm{E}-22$ & $3.463142 \mathrm{E}-07$ & $1.760880 \mathrm{E}-07$ \\
4: $16 \times 60$ & $4.601885 \mathrm{E}-22$ & $2.710648 \mathrm{E}-22$ & $2.951471 \mathrm{E}-07$ & $1.351625 \mathrm{E}-07$ \\
5: $32 \times 124$ & $3.490756 \mathrm{E}-22$ & $2.488107 \mathrm{E}-22$ & $1.751641 \mathrm{E}-07$ & $5.954642 \mathrm{E}-08$ \\
6: $64 \times 252$ & $3.273669 \mathrm{E}-22$ & $1.955235 \mathrm{E}-22$ & $9.366299 \mathrm{E}-08$ & $2.821412 \mathrm{E}-08$ \\
7: $128 \times 508$ & $2.354253 \mathrm{E}-22$ & $1.492452 \mathrm{E}-22$ & $4.831483 \mathrm{E}-08$ & $1.368716 \mathrm{E}-08$ \\
8: $256 \times 1020$ & $2.955694 \mathrm{E}-22$ & $4.467785 \mathrm{E}-22$ & $2.452613 \mathrm{E}-08$ & $6.727131 \mathrm{E}-09$ \\
9: $512 \times 2044$ & $2.445121 \mathrm{E}-22$ & $3.411378 \mathrm{E}-22$ & $1.235035 \mathrm{E}-08$ & $3.334926 \mathrm{E}-09$ \\
10: $1024 \times 4092$ & $1.869314 \mathrm{E}-22$ & $1.980601 \mathrm{E}-22$ & $6.196520 \mathrm{E}-09$ & $1.659675 \mathrm{E}-09$ \\
11: $2048 \times 8188$ & $2.635941 \mathrm{E}-22$ & $1.341339 \mathrm{E}-21$ & $3.103560 \mathrm{E}-09$ & $8.277541 \mathrm{E}-10$ \\
12: $4096 \times 16380$ & $1.793038 \mathrm{E}-22$ & $4.370611 \mathrm{E}-22$ & $1.930379 \mathrm{E}-09$ & $2.611492 \mathrm{E}-11$ \\
\hline
\end{tabular}

Table 2. Spherical Analysis and Synthesis of 11 Resolution Levels of Octahedral Simulation Using the Least-Squares Formulation on the Unit Sphere

\begin{tabular}{lcccc}
\hline OCTAHEDRAL & \multicolumn{2}{c}{ LS SHT of EGM2008 without mask } & \multicolumn{2}{c}{ LS SHT of EGM2008 with mask } \\
\hline Level: Grid & Spectral RMS & Spatial RMS & Spectral RMS & Spatial RMS \\
\hline \hline $1: 2 \times 4$ & $0.000000 \mathrm{E}+00$ & $0.000000 \mathrm{E}+00$ & $0.000000 \mathrm{E}+00$ & $0.000000 \mathrm{E}+00$ \\
2: $4 \times 12$ & $1.697587 \mathrm{E}-22$ & $5.946380 \mathrm{E}-22$ & $4.372804 \mathrm{E}-07$ & $1.477959 \mathrm{E}-06$ \\
3: $8 \times 28$ & $1.997728 \mathrm{E}-22$ & $1.728191 \mathrm{E}-21$ & $2.818574 \mathrm{E}-07$ & $2.039057 \mathrm{E}-06$ \\
4: $16 \times 60$ & $1.313014 \mathrm{E}-22$ & $2.137550 \mathrm{E}-21$ & $1.695670 \mathrm{E}-07$ & $1.822784 \mathrm{E}-06$ \\
5: $32 \times 124$ & $1.121353 \mathrm{E}-22$ & $3.273131 \mathrm{E}-21$ & $9.212659 \mathrm{E}-08$ & $1.767461 \mathrm{E}-06$ \\
6: $64 \times 252$ & $7.957850 \mathrm{E}-23$ & $4.512217 \mathrm{E}-21$ & $4.791357 \mathrm{E}-08$ & $1.732168 \mathrm{E}-06$ \\
7: $128 \times 508$ & $5.157020 \mathrm{E}-23$ & $5.277815 \mathrm{E}-21$ & $2.442382 \mathrm{E}-08$ & $1.712134 \mathrm{E}-06$ \\
$8: 256 \times 1020$ & $3.448122 \mathrm{E}-23$ & $7.576252 \mathrm{E}-21$ & $1.232450 \mathrm{E}-08$ & $1.702431 \mathrm{E}-06$ \\
9: $512 \times 2044$ & $2.723784 \mathrm{E}-23$ & $1.145658 \mathrm{E}-20$ & $6.190027 \mathrm{E}-09$ & $1.696969 \mathrm{E}-06$ \\
10: $1024 \times 4092$ & $1.826069 \mathrm{E}-23$ & $1.496024 \mathrm{E}-20$ & $3.101933 \mathrm{E}-09$ & $1.693970 \mathrm{E}-06$ \\
$11: 2048 \times 8188$ & $1.271623 \mathrm{E}-23$ & $2.125242 \mathrm{E}-20$ & $1.552696 \mathrm{E}-09$ & $1.692318 \mathrm{E}-06$ \\
\hline
\end{tabular}

Blais, J.A.R. and D.A. Provins: Spherical Harmonic Analysis and Synthesis for Global Multiresolution Applications. Journal of Geodesy, vol. 76, no. 1, pp. 29--35, 2002.

Blais, J.A.R. and Z. Zhang: Exploring Pseudo- and Chaotic Random Monte Carlo Simulations. Computers and Geosciences (2011), doi:10.1016/j.cageo.2011.01.009, Elsevier Ltd.

Colombo, O.L.: Numerical Methods for Harmonic Analysis on the Sphere. Report no. 310, Department of Geodetic Science and Surveying, The Ohio State University, 1981.

Cui, J.: Finite Pointset Methods on the Sphere and Their Application in Physical Geodesy. Ph.D. thesis, 1995, University of Kaiserlautern, Geomathematics Group, Germany.

Cui, J. and W. Freeden: Equidistribution on the Sphere. SIAM Journal of Scientific Computations, Vol. 18, No. 2, pp. 595--609, 1997.
Flury, J.: Short-Wavelength Spectral Properties of the Gravity Field from a Range of Regional Data Sets. Journal of Geodesy, 79, 624--640, 2006.

Freeden, W.: Constructive Approximation on the Sphere with Applications to Geomathematics. 427 pages, 1998, Clarendon Press.

Gorski K.M., Hivon E., Banday A.J., Wandelt B.D., Hansen F.K., Reinecke M. and Bartelmann M., HEALPix: A Framework for High Resolution Discretization and Fast Analysis of Data Distributed on the Sphere. The Astronomical Journal, 622(2):759--771, 2005.

Haagmans, R.H.: A Synthetic Earth for Geodesy. Journal of Geodesy, 74, pp. 503--511, 2000.

Heiskanen, W.A. and H. Moritz: Physical Geodesy, W.H. Freeman and Company, San Francisco, 363pp, 1967. 
Table 3. Spherical Analysis and Synthesis of 10 Resolution Levels of Icosahedral Simulation Using the Chebychev Quadrature Formulation on the Unit Sphere

\begin{tabular}{lcccc}
\hline ICOSAHEDRAL & CQ SHT of EGM2008 without mask & \multicolumn{2}{c}{ CQ SHT of EGM2008 with mask } \\
\hline Level: Grid & Spectral RMS & Spatial RMS & Spectral RMS & Spatial RMS \\
\hline \hline 2: $6 \times 20$ & $5.804421 \mathrm{E}-22$ & $3.089255 \mathrm{E}-22$ & $5.169650 \mathrm{E}-08$ & $2.974115 \mathrm{E}-08$ \\
3: $12 \times 40$ & $5.365887 \mathrm{E}-22$ & $3.109832 \mathrm{E}-22$ & $2.349163 \mathrm{E}-07$ & $1.178687 \mathrm{E}-07$ \\
4: $24 \times 80$ & $5.341677 \mathrm{E}-22$ & $4.003079 \mathrm{E}-22$ & $1.488747 \mathrm{E}-07$ & $6.880287 \mathrm{E}-08$ \\
5: $48 \times 160$ & $3.906096 \mathrm{E}-22$ & $2.792877 \mathrm{E}-22$ & $8.434646 \mathrm{E}-08$ & $2.841566 \mathrm{E}-08$ \\
6: $96 \times 320$ & $2.393798 \mathrm{E}-22$ & $1.717077 \mathrm{E}-22$ & $4.363152 \mathrm{E}-08$ & $1.329424 \mathrm{E}-08$ \\
7: $192 \times 640$ & $2.348442 \mathrm{E}-22$ & $2.536293 \mathrm{E}-22$ & $2.218180 \mathrm{E}-08$ & $6.423378 \mathrm{E}-09$ \\
8: $384 \times 1280$ & $3.764144 \mathrm{E}-22$ & $7.514292 \mathrm{E}-22$ & $1.117999 \mathrm{E}-08$ & $3.140013 \mathrm{E}-09$ \\
9: $768 \times 2560$ & $2.441085 \mathrm{E}-22$ & $5.553739 \mathrm{E}-22$ & $5.611133 \mathrm{E}-09$ & $1.552097 \mathrm{E}-09$ \\
10: $1536 \times 5120$ & $2.102354 \mathrm{E}-22$ & $7.987596 \mathrm{E}-22$ & $2.810755 \mathrm{E}-09$ & $7.712216 \mathrm{E}-10$ \\
11: $3072 \times 10240$ & $2.673822 \mathrm{E}-22$ & $1.172834 \mathrm{E}-21$ & $1.406672 \mathrm{E}-09$ & $3.843184 \mathrm{E}-10$ \\
\hline
\end{tabular}

Table 4. Spherical Analysis and Synthesis of 10 Resolution Levels of Icosahedral Simulation Using the Least-Squares Formulation on the Unit Sphere

\begin{tabular}{lcccc}
\hline ICOSAHEDRAL & \multicolumn{2}{c}{ LS SHT of EGM2008 without mask } & \multicolumn{2}{c}{ LS SHT of EGM2008 with mask } \\
\hline Level: Grid & Spectral RMS & Spatial RMS & Spectral RMS & Spatial RMS \\
\hline \hline 1: $3 \times 10$ & $1.252378 \mathrm{E}-22$ & $2.117582 \mathrm{E}-22$ & $1.252378 \mathrm{E}-22$ & $2.593498 \mathrm{E}-22$ \\
2: $6 \times 20$ & $2.951397 \mathrm{E}-22$ & $1.510085 \mathrm{E}-21$ & $2.510529 \mathrm{E}-07$ & $1.465168 \mathrm{E}-06$ \\
3: $12 \times 40$ & $1.524520 \mathrm{E}-22$ & $2.203792 \mathrm{E}-21$ & $1.529617 \mathrm{E}-07$ & $1.681240 \mathrm{E}-06$ \\
4: $24 \times 80$ & $1.155720 \mathrm{E}-22$ & $2.779775 \mathrm{E}-21$ & $8.480966 \mathrm{E}-08$ & $1.357296 \mathrm{E}-06$ \\
5: $48 \times 160$ & $1.058833 \mathrm{E}-22$ & $4.664491 \mathrm{E}-21$ & $4.372940 \mathrm{E}-08$ & $1.274377 \mathrm{E}-06$ \\
6: $96 \times 320$ & $5.348252 \mathrm{E}-23$ & $4.455022 \mathrm{E}-21$ & $2.220202 \mathrm{E}-08$ & $1.231983 \mathrm{E}-06$ \\
7: $192 \times 640$ & $4.069242 \mathrm{E}-23$ & $6.624655 \mathrm{E}-21$ & $1.118425 \mathrm{E}-08$ & $1.205037 \mathrm{E}-06$ \\
8: $384 \times 1280$ & $3.885973 \mathrm{E}-23$ & $1.155375 \mathrm{E}-20$ & $5.612149 \mathrm{E}-09$ & $1.191590 \mathrm{E}-06$ \\
9: $768 \times 2560$ & $2.022858 \mathrm{E}-23$ & $1.247948 \mathrm{E}-20$ & $2.810998 \mathrm{E}-09$ & $1.184380 \mathrm{E}-06$ \\
10: $1536 \times 5120$ & $1.586258 \mathrm{E}-23$ & $1.930839 \mathrm{E}-20$ & $1.406731 \mathrm{E}-09$ & $1.180518 \mathrm{E}-06$ \\
\hline
\end{tabular}

Fekete, G. and L. Treinish: Sphere Quadtrees: A New Data Structure to Support the Visualization of Spherically Distributed Data. SPIE, Extracting Meaning from Complex Data: Processing, Display, Interaction, Vol. 1259, pp. 242--250, 1990.

Klees, R., M. Van Gelderen, C. Lage and C. Schwab: Fast Numerical Solution of the Linearized Molodensky Problem. Journal of Geodesy, 75, pp. 349--362, 2001.

Morokoff, W. and R.E. Caflisch: Quasi-random sequences and their discrepancies. SIAM J. Sci. Comput., 15, pp. 1251--1279, 1994.
Pavlis N.K., Holmes S.A., Kenyon S.C. and Factor J.K., An Earth Gravitational Model to Degree 2160: EGM2008. Presentation at the 2008 General Assembly of the European Geosciences Union, Vienna, Austria, April 13--18, 2008.

Rapp, R.H.: The Decay of the Spectrum of the Gravitational Potential and the Topography of the Earth. Geophys. J. Int. 99, 449--455, 1989.

Sneeuw, N.: Global Spherical Harmonic Analysis by Least-Squares and Numerical Quadrature Methods in Historical Perspective. Geophys. J. Int. 118, 707--716, 1994. 